\title{
SYNTHESIS AND ANTICANCER ACTIVITY EVALUATION OF NOVEL DERIVATIVES OF 7-AMINO-4-METHYLQUINOLIN-2(1H)-ONE
}

\author{
KRZYSZTOF P. KUBICA ${ }^{1 *}$, PRZEMYSLAW P. TACIAK ${ }^{2}$, AGNIESZKA CZAJKOWSKA $^{3}$, \\ ALICJA SZTOKFISZ-IGNASIAK ${ }^{4}$, RAFAL WYREBIAK ${ }^{5}$, IZABELA MLYNARCZUK-BIALY4, \\ JACEK MALEJCZYK ${ }^{4}$ and ALEKSANDER P. MAZUREK ${ }^{1,6}$
}
${ }^{1}$ Department of Drug Chemistry, Medical University of Warsaw, Banacha 1, 02-097 Warsaw, Poland ${ }^{2}$ Department of Pharmacodynamics, Center of Preclinical Research and Technology, Żwirki i Wigury 81, 02-091 Warsaw, Poland ${ }^{3}$ Department of Transplantology and Central Tissue Bank, Center of Biostructure Research, Medical University of Warsaw, Chałubińskiego 5, 02-004 Warsaw, Poland
${ }^{4}$ Department of Histology and Embryology, Center of Biostructure Research, Medical University of Warsaw, Chałubińskiego 5, 02-004 Warsaw, Poland ${ }^{5}$ Department of Biomaterials Chemistry, Chair of Inorganic and Analytical Chemistry, Medical University of Warsaw, Banacha 1, 02-097 Warsaw, Poland ${ }^{6}$ National Medicines Institute, Chełmska 30/34, 00-725 Warsaw, Poland

\begin{abstract}
In this study, we designed and synthesized sixteen new derivatives of 7-amino-4-methylquinolin$2(1 \mathrm{H})$-one with potential anticancer activity. The structures of synthesized compounds were confirmed by $1 \mathrm{H}$ and ${ }^{13} \mathrm{C}$ NMR. The activity of novel substances was evaluated by cell viability assay and wound healing assay. In vitro tests for series of sixteen novel compounds were performed. The results showed that examined compounds are selective for a cancer cells, but their activity for various types of cancer is different. Three of the new compounds presented the ability to inhibit cells migration. The novel compounds constitute a good starting point for further studies and optimization of structure for new therapeutically effective anti-cancerous drugs. Seven compounds, which showed the highest rate of cell inhibition, were selected for further studies.
\end{abstract}

Keywords: anticancer activity, cytotoxicity assay, cell migration assay, quinolin-2(1H)-one

It is estimated, that in a few years cancer will be the most common cause of death worldwide (1, 2). Therefore, there is a need for development of new substances with potential anticancer activity.

In our study, we designed a series of new compounds, shown in Scheme 1, with a high correlation with structures of several drugs used in anticancer therapy, belonging to the following classes of drugs: Inhibitors of protein kinases. The process of cancerogenesis may depend on external and certain genetic factors. In some types of neoplasia, e.g. Chronic Myeloid Leukemia, expression of specific kinases is one of the basic factors of oncogenic transformation. Imatinib (Glivec) became a "gold standard" in the treatment of patients with Chronic Myeloid Leukemia owing to selective inhibition of the tyrosine kinase what is leading to high efficacy and good tolerability (3). Cab is already used for treatment of the patients with medullary thyroid cancer (4) and kidney cancer (5). Other drugs are in clinical trials stage (6). In scheme 1 we can see structural compliance with Imatinib. It is worth to pay attention to the quinoline moiety, which is very common in the group of anticancer drugs that are inhibitors of protein kinases.

Folic acid antagonists. Antifolates are a group of drugs that use the properties of folic acid as one of the most important coenzymes in the synthesis of nucleic acids, RNA and DNA. These drugs are most effective in cells with high production of nucleic acids, which are rapidly proliferating (among others tumor cells). The protected hydroxyl groups indicate a structural correlation with trimetrexate, which is a folic acid antagonist $(7,8)$.

We also decided to unblock compounds with protected hydroxyl groups. Thus, we obtained com-

\footnotetext{
* Corresponding author: e-mail: krzysztof.pawel.kubica@gmail.com
} 
pounds, which show analogy with phenolic acids. Naturally occurring polyphenols show high antioxidant activity. The structures can serve as a base for creating new molecules with anticancer activity (9, 10). Obtained compounds show high similarity with coumarin esters of gallic acid which appeared to have anticancer activity $(10,11)$.

\section{EXPERIMENTAL}

\section{CHEMICAL METHODOLOGY}

The main goal of this work was to synthesize the sixteen new derivatives of 7-amino-4methylquinolin-2(1H)-one. Firstly, the phenol groups of phenolic acids were protected by acylation with acetic anhydride. Then, protected compounds were converted to acid chlorides using thionyl chloride. The final amides were obtained by reacting protected acid chlorides with 7-amino-4-methylquinolin-2(1H)-one in dichloromethane to give novel, non-reported yet compounds. In final reaction, trimethylamine was added as an acceptor of hydrogen chloride. The structures of synthesized compounds were confirmed by ${ }^{1} \mathrm{H}$ and ${ }^{13} \mathrm{C}$ NMR.
All chemicals were purchased from major chemical suppliers, were of high or highest purity grade and used without any further purification. Melting points were determined in open glass capillaries with MEL-TEMP II apparatus (Laboratory Devices, USA) and were uncorrected. ${ }^{1} \mathrm{H}$ and ${ }^{13} \mathrm{C}$ NMR spectra in solution were recorded at $25^{\circ} \mathrm{C}$ with a Bruker AVANCE III HD (500 MHz). The calculated shielding constants were used as an aid in assignment of resonances of ${ }^{13} \mathrm{C}$ atoms. Chemical $\delta$ shifts (ppm) were referred to tetramethylsilane. The purity of the compounds was routinely checked by thin layer chromatography (TLC) using Kieselgel 60 $\mathrm{F}_{254}$ sheets. Spots were detected under UV light.

The 7-amino-4-methylquinolin-2(1H)-one $\mathbf{1}$ was synthesized according to the procedure described in the literature (12). The new compounds 2-17 were synthesized according to the procedures showed below.

\section{Exemplary procedure for preparation of amides (II) (2-7)}

7-amino-4-methylquinolin-2(1H)-one $\quad(1.00$ eq) was dissolved in dichloromethane and stirred for

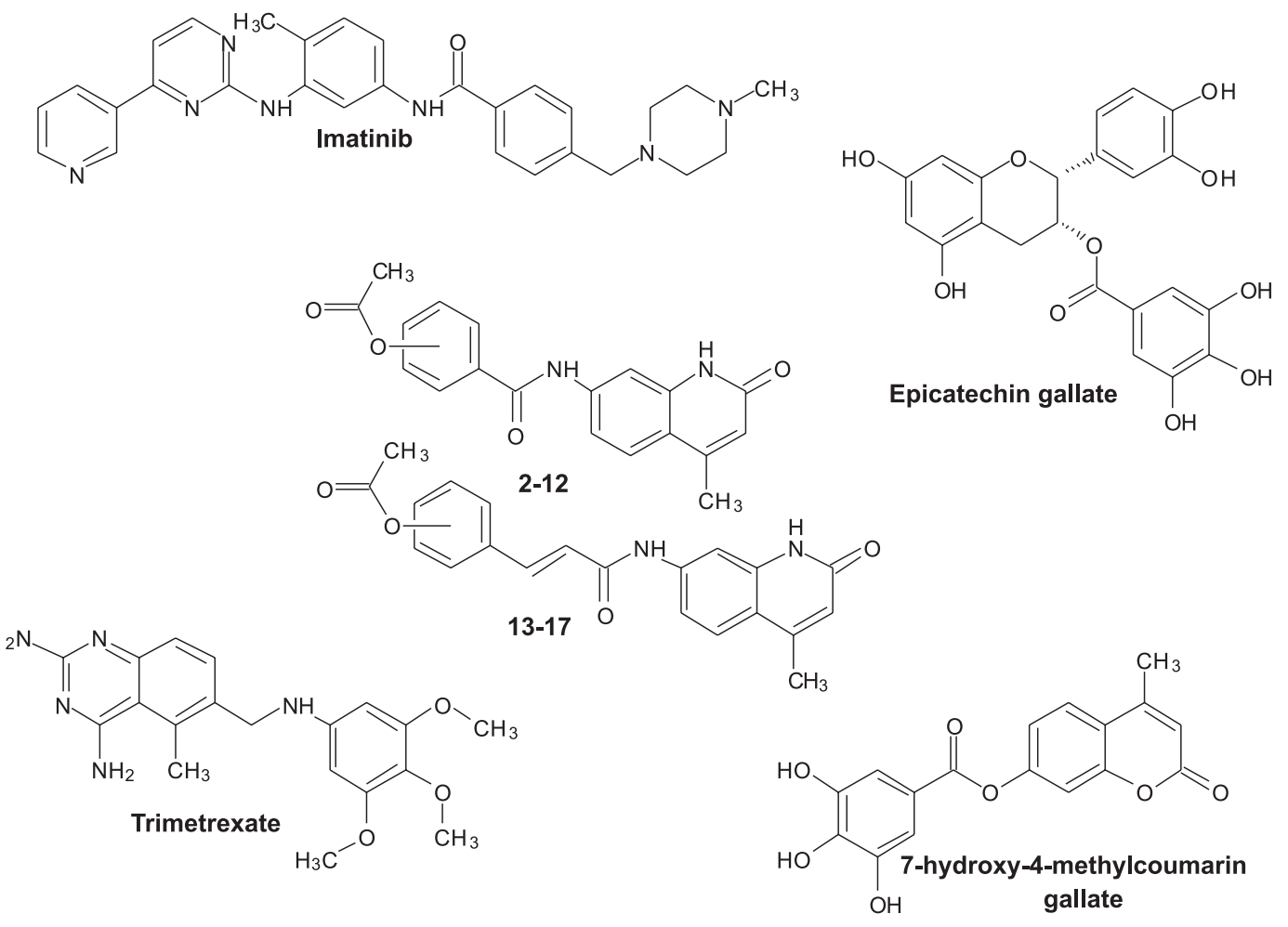

Scheme 1. Structural compliance between new designed derivatives and anticancers agents 
15 min at ambient temperature, meanwhile, the triethylamine (1.05 eq) was added. After $15 \mathrm{~min}$ the proper benzoyl chloride (1.05 eq) was added dropwise (benzoyl chloride for compound 2; 3-acetoxybenzoyl chloride for compound 3; 4-acetoxybenzoyl chloride for compound 4; 3,4-diacetoxybenzoyl chloride for compound 5; 3,5-diacetoxybenzoyl chloride for compound 6; 3,4,5-triacetoxybenzoyl chloride for compound 7). The course of the reaction was monitored by TLC. After 15 min product was filtrated and recrystallized from $\mathrm{CH}_{2} \mathrm{Cl}_{2}:\left(\mathrm{CH}_{3}\right)_{2} \mathrm{CO} 1: 1$.

\section{Exemplary procedure for preparation of amides (III) (13-15)}

7-amino-4-methylquinolin-2(1H)-one (1.00eq) was dissolved in dichloromethane and stirred for 15 min at ambient temperature, meanwhile the triethylamine (1.05 eq) was added. After $15 \mathrm{~min}$ the proper cinnamoyl chloride (1.05 eq) was added dropwise (cinnamoyl chloride for compound 13; 3,4-diacetoxycinnamoyl chloride for compound 14; 3 methoxy-4-acetoxycinnamoyl chloride for compound 15). The course of the reaction was monitored by TLC. After $15 \mathrm{~min}$ product was filtrated and recrystallized from $\mathrm{CH}_{2} \mathrm{Cl}_{2}:\left(\mathrm{CH}_{3}\right)_{2} \mathrm{CO} 1: 1$.

\section{Exemplary procedure for deprotection (IV) (8-12 \& 16-17)}

The proper amide (1.00 eq) was dissolved in dichloromethane and stirred for $15 \mathrm{~min}$ at ambient temperature, then the $1 \mathrm{M} \mathrm{NaOH}$ (4.00 eq) was added. After $45 \mathrm{~min}$ the acetic acid (8.00 eq) was added dropwise. The course of the reaction was monitored by TLC. After 15 min product was filtered and recrystallized from $\mathrm{CH}_{2} \mathrm{Cl}_{2}:\left(\mathrm{CH}_{3}\right)_{2} \mathrm{CO} 1: 1$.

\section{7-N-benzoylamino-4-methylquinolin-2(1H)-one (2)}

The title compound was obtained in $93 \%$ yield, starting from 1 ; m.p. $310^{\circ} \mathrm{C}$;

${ }^{1} \mathrm{H}$ NMR (500 MHz, DMSO-d $\left.\mathrm{d}_{\mathrm{d}}\right) \delta 11.67$ (br s, $1 \mathrm{H}, \mathrm{H}-1), 10.55$ (s, 1H, 7-NH), 8.04 (d, $J=1.3 \mathrm{~Hz}$, $1 \mathrm{H}, \mathrm{H}-8), 8.01$ (d, $J=7.4 \mathrm{~Hz}, 2 \mathrm{H}, \mathrm{H}-2$ ' and H-6'), $7.68(\mathrm{~d}, J=8.7 \mathrm{~Hz}, 1 \mathrm{H}, \mathrm{H}-6), 7.62(\mathrm{t}, J=7.2 \mathrm{~Hz}, 1 \mathrm{H}$, H-4'), $7.59-7.52$ (m, 3H, H-3', H-5' and H-5), 6.31 (s, $1 \mathrm{H}, \mathrm{H}-3), 2.41$ (s, 3H, 4- $\left.\mathrm{CH}_{3}\right) ;{ }^{13} \mathrm{C}$ NMR (126 MHz, DMSO- $\mathrm{d}_{6}$ ) $\delta 165.85$ (amide $\mathrm{C}=\mathrm{O}$ ), 162.07 (C2), 147.67, 140.94, 139.33, 134.68, 131.74, 128.37, $127.78,125.08,119.16,115.85,114.66$ and 105.71 (aromatic), $18.39\left(4-\mathrm{CH}_{3}\right)$.

\section{7-N-(3-acetoxybenzoyl)-amino-4-methylquinolin-} 2(1H)-one (3)

The title compound was obtained in $88 \%$ yield, starting from $1 ;$ m.p. $305^{\circ} \mathrm{C}$;
${ }^{1} \mathrm{H}$ NMR (500 MHz, DMSO-d $\left.{ }_{6}\right) \delta 11.66$ (br s, 1H, H-1), 10.57 (s, 1H, 7-NH), 8.02 (d, $J=1.8 \mathrm{~Hz}$, $1 \mathrm{H}, \mathrm{H}-8), 7.92$ (d, J = 7.8 Hz, 1H, H-4'), 7.76 (s, 1H, H-2'), 7.69 (d, $J=8.8 \mathrm{~Hz}, 1 \mathrm{H}, \mathrm{H}-5), 7.61$ (t, $J=7.9$ $\mathrm{Hz}, 1 \mathrm{H}, \mathrm{H}-5$ ') 7.55 (dd, $J=8.8,1.9 \mathrm{~Hz}, 1 \mathrm{H}, \mathrm{H}-6$ ), 7.40 (dd, $J=8.0,1.6 \mathrm{~Hz}, 1 \mathrm{H}, \mathrm{H}-6$ '), 6.31 (s, 1H, H3), 2.41 (s, 3H, 4- $\left.\mathrm{CH}_{3}\right), 2.33$ (s, 3H, acetoxy $\left.-\mathrm{CH}_{3}\right)$; ${ }^{13} \mathrm{C}$ NMR (126 MHz, DMSO-d $\left.\mathrm{d}_{6}\right) \delta 169.18$ (acetoxy $\mathrm{C}=\mathrm{O}$ ), 164.75 (amide $\mathrm{C}=\mathrm{O}$ ), 162.04 (C-2), 150.39, $147.62,140.70,139.33,136.05,129.59,125.39$, $125.22,125.12,121.21,119.28,115.96,114.64$ and 105.80 (aromatic), 20.81 (acetoxy $-\mathrm{CH}_{3}$ ), 18.38 (4$\left.\mathrm{CH}_{3}\right)$.

7-N-(4-acetoxybenzoyl)-amino-4-methylquinolin2(1H)-one (4)

The title compound was obtained in $86 \%$ yield, starting from 1 ; m.p. $335^{\circ} \mathrm{C}$;

${ }^{1} \mathrm{H}$ NMR (500 MHz, DMSO-d $\left.{ }_{6}\right) \delta 11.64$ (br s, $1 \mathrm{H}, \mathrm{H}-1), 10.54$ (s, 1H, 7-NH), 8.03 (dt, $J=8.5,2.3$ Hz, 2H, H-3' and H-5'), 8.00 (d, $J=2.0 \mathrm{~Hz}, 1 \mathrm{H}, \mathrm{H}-$ 8), 7.67 (d, $J=8.8 \mathrm{~Hz}, 1 \mathrm{H}, \mathrm{H}-5), 7.53$ (dd, $J=9.0$, $2.0 \mathrm{~Hz}, 1 \mathrm{H}, \mathrm{H}-6), 7.30$ (dt, $J=9.0,2.3 \mathrm{~Hz}, 2 \mathrm{H}, \mathrm{H}-2$ ' and H-6'), 6.29 (d, $J=0.7 \mathrm{~Hz}, 1 \mathrm{H}, \mathrm{H}-3), 2.39$ (d, $J$ $\left.=0.6 \mathrm{~Hz}, 3 \mathrm{H}, 4-\mathrm{CH}_{3}\right), 2.31\left(\mathrm{~s}, 3 \mathrm{H}\right.$, acetoxy $\left.-\mathrm{CH}_{3}\right)$; ${ }^{13} \mathrm{C}$ NMR (126 MHz, DMSO-d $\mathrm{d}_{6}$ ) $\delta 168.97$ (acetoxy $\mathrm{C}=\mathrm{O}$ ), 165.10 (amide $\mathrm{C}=\mathrm{O}$ ), 162.06 (C-2), 153.04, $147.68,140.88,139.33,132.20,129.35,125.11$, $121.84,115.89,114.60$ and 105.67 (aromatic), 20.86 (acetoxy $\left.-\mathrm{CH}_{3}\right), 18.39\left(4-\mathrm{CH}_{3}\right)$.

\section{7-N-(3,4-diacetoxybenzoyl)-amino-4-methylqui- nolin-2(1H)-one (5)}

The title compound was obtained in $92 \%$ yield, starting from 1; m.p. $297^{\circ} \mathrm{C}$;

${ }^{1} \mathrm{H}$ NMR (500 MHz, DMSO-d ${ }_{6}$ ) $\delta 11.64$ (br s, $1 \mathrm{H}, \mathrm{H}-1), 10.58$ (s, 1H, 7-NH), 7.98 (d, $J=1.3 \mathrm{~Hz}$, 1H, H-8), 7.95 (dd, $J=8.5,1.7$ Hz, 1H, H-6'), 7.90 (d, $J=1.7 \mathrm{~Hz}, 1 \mathrm{H}, \mathrm{H}-2$ ') 7.68 (d, $J=8.9 \mathrm{~Hz}, 1 \mathrm{H}$, H-5'), 7.52 (dd, $J=8.9,1.4 \mathrm{~Hz}, 1 \mathrm{H}, \mathrm{H}-6), 7.46$ (d, $J=8.5 \mathrm{~Hz}, 1 \mathrm{H}, \mathrm{H}-5), 6.30$ (s, 1H, H-3), 2.39 (s, 3H, 4- $\left.\mathrm{CH}_{3}\right), 2.33\left(\mathrm{~s}, 3 \mathrm{H}\right.$, acetoxy $\left.-\mathrm{CH}_{3}\right), 2.32(\mathrm{~s}, 3 \mathrm{H}$, acetoxy $\left.-\mathrm{CH}_{3}\right) ;{ }^{13} \mathrm{C}$ NMR $\left(126 \mathrm{MHz}, \mathrm{DMSO}-\mathrm{d}_{6}\right) \delta$ 168.25 and 168.04 (acetoxy $\mathrm{C}=\mathrm{O}$ ), 164.23 (amide $\mathrm{C}=\mathrm{O}), 162.08 \quad(\mathrm{C}-2), 147.66,144.78,141.88$, $140.72,139.37,133.18,126.37,125.17,123.78$, $123.35,119.33,116.02,114.64$ and 105.81 (aromatic), 20.40 and 20.33 (acetoxy $-\mathrm{CH}_{3}$ ), 18.41 (4$\mathrm{CH}_{3}$ ).

\section{7-N-(3,5-diacetoxybenzoyl)-amino-4-methylqui- nolin-2(1H)-one $(6)$}

The title compound was obtained in $90 \%$ yield, starting from 1 ; m.p. $276^{\circ} \mathrm{C}$; 
${ }^{1} \mathrm{H}$ NMR (500 MHz, DMSO-d $\left.{ }_{6}\right) \delta 11.70$ (br s, $1 \mathrm{H}, \mathrm{H}-1), 10.63$ (s, 1H, 7-NH), 8.00 (d, $J=2.0 \mathrm{~Hz}$, $1 \mathrm{H}, \mathrm{H}-8), 7.72$ (d, $J=2.1 \mathrm{~Hz}, 2 \mathrm{H}, \mathrm{H}-2^{\prime}$ and H-6'), $7.70(\mathrm{~d}, J=8.8 \mathrm{~Hz}, 1 \mathrm{H}, \mathrm{H}-5), 7.55(\mathrm{dd}, J=8.8,2.1$ Hz, 1H, H-6), 7.31 (t, $J=2.1 \mathrm{~Hz}, 1 \mathrm{H}, \mathrm{H}-4$ '), 6.32 (d, $J=1.0 \mathrm{~Hz}, 1 \mathrm{H}, \mathrm{H}-3), 2.41(\mathrm{~d}, J=0.9 \mathrm{~Hz}, 3 \mathrm{H}, 4-$ $\left.\mathrm{CH}_{3}\right), 2.33$ (s, 6H acetoxy $\left.-\mathrm{CH}_{3}\right) ;{ }^{13} \mathrm{C}$ NMR (126 $\mathrm{MHz}, \mathrm{DMSO}-\mathrm{d}_{6}$ ) $\delta 168.95$ (acetoxy $\mathrm{C}=\mathrm{O}$ ), 163.80 (amide $\mathrm{C}=\mathrm{O}$ ), 162.03 (C-2), 150.75, 147.71, 140.53, $139.28,136.53,125.15,119.55,119.28,118.85$, $116.09,114.72$ and 105.91 (aromatic), 20.7733 (acetoxy $\left.-\mathrm{CH}_{3}\right), 18.39\left(4-\mathrm{CH}_{3}\right)$.

7-N-(3,4,5-triacetoxybenzoyl)-amino-4-methylquinolin-2(1H)-one (7)

The title compound was obtained in $85 \%$ yield, starting from 1; m.p. $280^{\circ} \mathrm{C}$;

${ }^{1} \mathrm{H}$ NMR (500 MHz, DMSO-d $\left.{ }_{6}\right) \delta 11.63$ (br s, $1 \mathrm{H}, \mathrm{H}-1), 10.59$ (s, 1H, 7-NH), 7.95 (d, $J=1.9 \mathrm{~Hz}$, 1H, H-8), 7.85 (s, 2H, H-2' and H-6'), 7.69 (d, $J=$ $8.8 \mathrm{~Hz}, 1 \mathrm{H}, \mathrm{H}-5), 7.50$ (dd, $J=8.8,2.0 \mathrm{~Hz}, 1 \mathrm{H}, \mathrm{H}-$ 6), $6.30(\mathrm{~s}, 1 \mathrm{H}, \mathrm{H}-3), 2.40(\mathrm{~d}, J=0.7 \mathrm{~Hz}, 3 \mathrm{H}, 4-$ $\mathrm{CH}_{3}$ ), 2.35 (s, 3H, 4' acetoxy $\left.-\mathrm{CH}_{3}\right), 2.33$ (s, 6H, 3' and 5' acetoxy $\left.-\mathrm{CH}_{3}\right) ;{ }^{13} \mathrm{C}$ NMR (126 MHz, DMSO$\left.\mathrm{d}_{6}\right) \delta 168.03$ and 166.98 (acetoxy $\mathrm{C}=\mathrm{O}$ ), 163.41 (amide $\mathrm{C}=\mathrm{O}), 162.01$ (C-2), 147.61, 143.13, 140.48, $139.34,137.47,132.66,125.21,120.70,119.42$, 116.10, 114.62 and 105.87 (aromatic), 20.33 and $19.88\left(\right.$ acetoxy $\left.-\mathrm{CH}_{3}\right), 18.40\left(4-\mathrm{CH}_{3}\right)$.

\section{7-N-(3-hydroxybenzoyl)-amino-4-methylquino- lin-2(1H)-one (8)}

The title compound was obtained in $81 \%$ yield, starting from 3; m.p. $345^{\circ} \mathrm{C}$;

${ }^{1} \mathrm{H}$ NMR $\left(500 \mathrm{MHz}, \mathrm{DMSO}-\mathrm{d}_{6}\right) \delta 11.63(\mathrm{~s}, 1 \mathrm{H}$, H-1), 10.45 (s, 1H, 7-NH), 9.82 (s, 1H, 3'-OH), 8.03 (s, 1H, H-8), 7.67 (d, $J=8.1 \mathrm{~Hz}, 1 \mathrm{H}, \mathrm{H}-5), 7.54$ (d, $J=7.7 \mathrm{~Hz}, 1 \mathrm{H}, \mathrm{H}-6), 7.48-7.23(\mathrm{~m}, 3 \mathrm{H})$ and 7.01 (d, $J=6.5 \mathrm{~Hz}, 1 \mathrm{H}, \mathrm{H}-2^{\prime}, \mathrm{H}-4$ ', H-5' and H-6'), 6.30 (s, 1H, H-3), 2.41 (s, 3H, 4- $\left.\mathrm{CH}_{3}\right) ;{ }^{13} \mathrm{C}$ NMR (126 MHz, DMSO-d ${ }_{6}$ ) $\delta 165.87$ (amide $\mathrm{C}=\mathrm{O}$ ), 162.07 (C2), 157.35 (C-3'), 147.65, 140.96, 139.32, 136.12, $129.44,125.05,119.13,118.69,118.31,115.79$, $114.63,114.60$ and 105.67 (aromatic), 18.38 (4$\mathrm{CH}_{3}$ ).

\section{7-N-(4-hydroxybenzoyl)-amino-4-methylquino- lin-2(1H)-one (9)}

The title compound was obtained in $82 \%$ yield, starting from 4 ; m.p. $373^{\circ} \mathrm{C}$;

${ }^{1} \mathrm{H}$ NMR (500 MHz, DMSO-d $)_{6} \delta 11.64(\mathrm{~s}, 1 \mathrm{H}$, H-1), 10.29 (s, 1H, 7-NH), 10.25 (br s, 1H, 4'-OH), $8.03(\mathrm{~d}, J=2.0 \mathrm{~Hz}, 1 \mathrm{H}, \mathrm{H}-8), 7.93(\mathrm{dt}, J=8.5 \mathrm{~Hz}$, $2.5 \mathrm{~Hz}, 2 \mathrm{H}, \mathrm{H}-3$ ' and H-5'), 7.68 (d, $J=8.8 \mathrm{~Hz}, 1 \mathrm{H}$, H-5), 7.55 (dd, $J=8.8,2.0 \mathrm{~Hz}, 1 \mathrm{H}, \mathrm{H}-6), 6.92$ (dt, $J$ $=8.5 \mathrm{~Hz}, 2.4 \mathrm{~Hz}, 2 \mathrm{H}, \mathrm{H}-2^{\prime}$ ' and H-6'), 6.31 (d, $J=0.6$ $\mathrm{Hz}, 1 \mathrm{H}, \mathrm{H}-3), 2.42$ (d, $\left.J=0.9 \mathrm{~Hz}, 3 \mathrm{H}, 4-\mathrm{CH}_{3}\right) ;{ }^{13} \mathrm{C}$ NMR (126 MHz, DMSO-d d $) \delta 165.36$ (amide $\mathrm{C}=\mathrm{O}$ ), 162.11 (C-2), 160.76 (C-4'), 147.69, 141.27, 139.33, 129.89 , 125.09, 124.99, 118.94, 115.58, 114.93, 114.63 and 105.52 (aromatic), $18.38\left(4-\mathrm{CH}_{3}\right)$.

\section{7-N-(3,4-dihydroxybenzoyl)-amino-4-methylqui- nolin-2(1H)-one (10)}

The title compound was obtained in $88 \%$ yield, starting from 5; m.p. $333^{\circ} \mathrm{C}$;

${ }^{1} \mathrm{H}$ NMR (500 MHz, DMSO-d $\left.{ }_{6}\right) \delta 11.56(\mathrm{~s}, 1 \mathrm{H}$, H-1), 10.18 (s, 1H, 7-NH), 9.62 (br s, 1H) and 9.26 (br s, 1H, 3'-OH and 4'-OH), 7.97 (d, $J=1.8 \mathrm{~Hz}$, $1 \mathrm{H}, \mathrm{H}-8), 7.64$ (d, $J=8.8 \mathrm{~Hz}, 1 \mathrm{H}, \mathrm{H}-5), 7.49$ (dd, $J$ $=8.8,1.8 \mathrm{~Hz}, 1 \mathrm{H}, \mathrm{H}-6), 7.41(\mathrm{~d}, J=1.9 \mathrm{~Hz}, 1 \mathrm{H}, \mathrm{H}-$ 5'), 7.38 (dd, $J=8.2,2.0 \mathrm{~Hz}, 1 \mathrm{H}, \mathrm{H}-6$ '), 6.84 (d, $J=$ $8.2 \mathrm{~Hz}, 1 \mathrm{H}, \mathrm{H}-2$ '), 6.26 (s, 1H, H-3), 2.39 (s, 3H, 4$\left.\mathrm{CH}_{3}\right) ;{ }^{13} \mathrm{C}$ NMR (126 MHz, DMSO-d 6 ) $\delta 165.50$ (amide C=O), 162.06 (C-2), 149.11, 147.63, 144.98, $141.33,139.36,125.60,124.97,119.88,118.95$, $115.51,114.89,114.59$ and 105.47 (aromatic), 18.40 $\left(4-\mathrm{CH}_{3}\right)$.

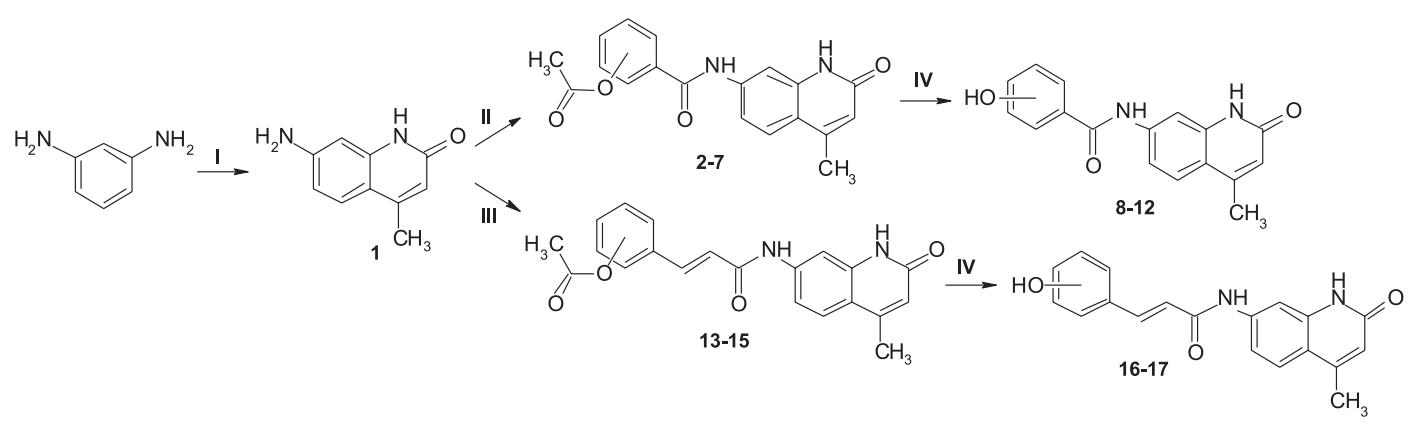

Figure 1. The synthesis of derivatives of 7-amino-1,2-dihydro-4 methyl-2-oxoquinoline. (I) Ethyl acetoacetate, pTSA, $185^{\circ} \mathrm{C}, 24 \mathrm{~h}$; (II \& III) Proper acid chloride, triethylamine, $\mathrm{CH}_{2} \mathrm{Cl}_{2}, 0.25 \mathrm{~h}$; (IV) $1.1 \mathrm{M} \mathrm{NaOH}, \mathrm{CH}_{2} \mathrm{Cl}_{2} 2 . \mathrm{CH}_{3} \mathrm{COOH}$ 
7-N-(3,5-dihydroxybenzoyl)-amino-4-methylquinolin-2(1H)-one (11)

The title compound was obtained in $82 \%$ yield, starting from 6; m.p. $373^{\circ} \mathrm{C}$;

${ }^{1} \mathrm{H}$ NMR (500 MHz, DMSO-d $)_{6} \delta 11.59(\mathrm{~s}, 1 \mathrm{H}$, H-1), 10.34 (s, 1H, 7-NH), 9.65 (br s, 2H, 3'-OH and 5 ' $-\mathrm{OH}), 8.00$ (s, 1H, H-8), 7.64 (d, $J=8.7 \mathrm{~Hz}$, $1 \mathrm{H}, \mathrm{H}-5), 7.49$ (d, $J=8.5 \mathrm{~Hz}, 1 \mathrm{H}, \mathrm{H}-6), 6.81$ (s, 2H, H-2' and H-6'), 6.44 (s, 1H, H-4'), 6.28 (s, 1H, H3), $2.38\left(\mathrm{~s}, 3 \mathrm{H}, 4-\mathrm{CH}_{3}\right) ;{ }^{13} \mathrm{C}$ NMR (126 MHz, $\mathrm{DMSO}_{6}$ ) $\delta 166.05$ (amide $\mathrm{C}=\mathrm{O}$ ), 162.07 (C-2), 158.34 (C-3' and C-5'), 147.67, 141.01, 139.30, $136.82,125.02,119.06,115.72,114.60,105.90$ and 105.61 (aromatic), $18.38\left(4-\mathrm{CH}_{3}\right)$.

7-N-(3,4,5-trihydroxybenzoyl)-amino-4-methylquinolin-2(1H)-one (12)

The title compound was obtained in $80 \%$ yield, starting from 7; m.p. $300^{\circ} \mathrm{C}$;

${ }^{1} \mathrm{H}$ NMR (500 MHz, DMSO-d $\left.{ }_{6}\right) \delta 11.54(\mathrm{~s}, 1 \mathrm{H}$, H-1), 10.15 (s, 1H, 7-NH), 9.17 (s, 2H, 3'-OH and 5'-OH), 8.84 (s, 1H, 4'-OH), 7.97 (d, $J=1.5 \mathrm{~Hz}$, $1 \mathrm{H}, \mathrm{H}-8), 7.63$ (d, $J=8.7 \mathrm{~Hz}, 1 \mathrm{H}, \mathrm{H}-5), 7.48$ (dd, $J$ $=8.7,1.5 \mathrm{~Hz}, 1 \mathrm{H}, \mathrm{H}-6), 6.98$ (s, 2H, H-2' and H-6'), $6.25(\mathrm{~s}, 1 \mathrm{H}, \mathrm{H}-3), 2.38\left(\mathrm{~s}, 3 \mathrm{H}, 4-\mathrm{CH}_{3}\right) ;{ }^{13} \mathrm{C} \mathrm{NMR}$ (126 MHz, DMSO-d d $_{6} \delta 165.80$ (amide $\mathrm{C}=\mathrm{O}$ ), 162.05 (C-2), 147.62, 145.49, 141.38, 139.36, $137.03,124.94,124.67,118.92,115.46,114.55$, 107.35 and 105.42 (aromatic), $18.40\left(4-\mathrm{CH}_{3}\right)$.

\section{7-N-cinnamoylamino-4-methylquinolin-2(1H)-} one (13)

The title compound was obtained in $90 \%$ yield, starting from 1 ; m.p. $375^{\circ} \mathrm{C}$;

${ }^{1} \mathrm{H}$ NMR (500 MHz, DMSO-d $\left.)_{6}\right) 11.60(\mathrm{~s}, 1 \mathrm{H}$, $\mathrm{H}-1), 10.47$ (s, 1H, 7-NH), 7.86 (d, $J=2.0 \mathrm{~Hz}, 1 \mathrm{H}$, $\mathrm{H}-8), 7.66$ (d, $J=8.8 \mathrm{~Hz}, 1 \mathrm{H}, \mathrm{H}-5), 7.65-7.59$ (m, $3 \mathrm{H}, \beta$ vinyl-H, H-6', H-2'), $7.49-7.39$ (m, 4H, H6, H-3', H-4', H-5'), 6.87 (d, $J=15.7 \mathrm{~Hz}, 1 \mathrm{H}, \alpha$ vinyl-H), 6.27 (s, 1H, H-3), 2.39 (d, $J=1.1 \mathrm{~Hz}, 3 \mathrm{H}$, 4- $\left.\mathrm{CH}_{3}\right) ;{ }^{13} \mathrm{C}$ NMR (126 MHz, DMSO-d $\left.\mathrm{d}_{6}\right) \delta 163.81$ (amide C=O), 162.02 (C-2), 147.61, 140.94, 140.67, $139.51,134.63,129.93,129.06,127.79,125.40$, $122.03,119.08,115.69,113.60$ and 104.58 (aromatic and vinyl), $18.39\left(4-\mathrm{CH}_{3}\right)$.

\section{7-N-(3,4-diacetoxycinnamoyl)-amino-4-methyl- quinolin-2(1H)-one (14)}

The title compound was obtained in $89 \%$ yield, starting from 1; m.p. $297^{\circ} \mathrm{C}$;

${ }^{1} \mathrm{H}$ NMR (500 MHz, DMSO-d $)_{6} \delta 11.60(\mathrm{~s}, 1 \mathrm{H}$, H-1), 10.50 (s, 1H, 7-NH), 7.85 (d, $J=2.0 \mathrm{~Hz}, 1 \mathrm{H}$, H-8), 7.66 (d, $J=8.8 \mathrm{~Hz}, 1 \mathrm{H}, \mathrm{H}-5), 7.61$ (d, $J=16.0$ $\mathrm{Hz}, 1 \mathrm{H}, \beta$ vinyl-H), 7.58 (dd, $J=8.5 \mathrm{~Hz}, 2.0 \mathrm{~Hz}, 1 \mathrm{H}$,
H-6'), 7.55 (d, $J=2.0 \mathrm{~Hz}, \mathrm{H}-5$ '), 7.45 (dd, $J=8.8$, $2.0 \mathrm{~Hz}, 1 \mathrm{H}, \mathrm{H}-6), 7.36$ (d, $J=8.3 \mathrm{~Hz}, 1 \mathrm{H}, \mathrm{H}-2$ '), 6.85 (d, $J=15.7 \mathrm{~Hz}, 1 \mathrm{H}, \alpha$ vinyl-H), $6.27(\mathrm{~s}, 1 \mathrm{H}, \mathrm{H}-3)$, $2.38\left(\mathrm{~d}, J=1.1 \mathrm{~Hz}, 3 \mathrm{H}, 4-\mathrm{CH}_{3}\right), 2.31(\mathrm{~s}, 3 \mathrm{H})$ and 2.30 (s, 3H, 3' and 4' acetoxy $\left.-\mathrm{CH}_{3}\right),{ }^{13} \mathrm{C}$ NMR (126 MHz, $\mathrm{DMSO}^{\left.-\mathrm{d}_{6}\right)} \delta 168.22$ and 168.16 (3' and 4' acetoxy $\mathrm{C}=\mathrm{O}$ ), 163.54 (amide $\mathrm{C}=\mathrm{O}), 162.01(\mathrm{C}-2), 147.61$, $143.01,142.35,140.86,139.50,138.92,133.52$, $126.11,125.43,124.28,123.17,122.60,119.12$, 115.75, 113.61 and 104.61 (aromatic and vinyl), 20.37 (3' and 4' acetoxy $\left.-\mathrm{CH}_{3}\right), 18.39\left(4-\mathrm{CH}_{3}\right)$.

7-N-(3-methoxy-4-acetoxycinnamoyl)-amino-4methylquinolin-2(1H)-one (15)

The title compound was obtained in $95 \%$ yield, starting from $\mathbf{1}$; m.p. $310^{\circ} \mathrm{C}$;

${ }^{1} \mathrm{H}$ NMR $\left(500 \mathrm{MHz}, \mathrm{DMSO}-\mathrm{d}_{6}\right) \delta 11.60(\mathrm{~s}, 1 \mathrm{H}$, H-1), 10.49 (s, 1H, 7-NH), 7.87 (d, $J=2.0 \mathrm{~Hz}, 1 \mathrm{H}, \mathrm{H}-$ 8), $7.66(\mathrm{~d}, J=8.8 \mathrm{~Hz}, 1 \mathrm{H}, \mathrm{H}-5), 7.62(\mathrm{~d}, J=15.7 \mathrm{~Hz}$, $1 \mathrm{H}, \beta$ vinyl-H), 7.45 (dd, $J=8.8,2.1 \mathrm{~Hz}, 1 \mathrm{H}, 7.45)$, 7.39 (d, $J=1.8 \mathrm{~Hz}, 1 \mathrm{H}, \mathrm{H}-2$ '), 7.24 (dd, $J=8.4,1.8$ Hz, 1H, H-6'), 7.17 (d, J = 8.1 Hz, 1H, H-6'), 6.86 (d, $J=15.7 \mathrm{~Hz}, 1 \mathrm{H}, \alpha$ vinyl-H), 6.27 (s, 1H, H-3), 3.85 (s, 3H, 3'- $\left.-\mathrm{OCH}_{3}\right), 2.38\left(\mathrm{~d}, J=1.1 \mathrm{~Hz}, 3 \mathrm{H}, 4-\mathrm{CH}_{3}\right)$, 2.27 (s, 3H, 4' acetoxy $\left.-\mathrm{CH}_{3}\right) ;{ }^{13} \mathrm{C} \mathrm{NMR}(126 \mathrm{MHz}$, DMSO- $_{6}$ ) $\delta 168.44$ (acetoxy $\mathrm{C}=\mathrm{O}$ ), 163.76 (amide $\mathrm{C}=\mathrm{O}), 162.02$ (C-2), 151.14, 147.60, 140.94, 140.52, $140.08,139.52,133.63,125.41,123.42,122.29$, $120.48,119.08,115.69,113.60,111.68$ and 104.58 (aromatic and vinyl), 55.81 (methoxy $-\mathrm{CH}_{3}$ ), 20.42 (acetoxy $\left.-\mathrm{CH}_{3}\right), 18.39\left(4-\mathrm{CH}_{3}\right)$.

\section{7-N-(3,4-dihydroxycinnamoyl)-amino-4-meth-} ylquinolin-2(1H)-one (16)

The title compound was obtained in $85 \%$ yield, starting from 14 ; m.p. $350^{\circ} \mathrm{C}$;

${ }^{1} \mathrm{H}$ NMR (500 MHz, DMSO-d $\left.{ }_{6}\right) \delta 11.53(\mathrm{~s}, 1 \mathrm{H}$, H-1), 10.33 (s, 1H, 7-NH), 9.00 (br s, 2H, 3'-OH and 4'-OH), $7.72(\mathrm{~d}, J=2.1 \mathrm{~Hz}, 1 \mathrm{H}, \mathrm{H}-8), 7.62$ (d, $J=14.7 \mathrm{~Hz}, 1 \mathrm{H}, \beta$ vinyl-H), $7.45-7.39$ (m, 2H, H5 and H-6), 6.71 (d, $J=2.1 \mathrm{~Hz}, 1 \mathrm{H}, \mathrm{H}-6$ ') 6.67 (d, $\left.J=8.1 \mathrm{~Hz}, 1 \mathrm{H}, \mathrm{H}-2^{\prime}\right), 6.62(\mathrm{dd}, J=8.2,2.1 \mathrm{~Hz}, 1 \mathrm{H}$, H-5'), 6.58 (d, $J=15.6 \mathrm{~Hz}, 1 \mathrm{H}, \alpha$ vinyl-H), 6.29 (s, $1 \mathrm{H}, \mathrm{H}-3), 2.33$ (d, $\left.J=1.0 \mathrm{~Hz}, 3 \mathrm{H}, 4-\mathrm{CH}_{3}\right) .{ }^{13} \mathrm{C} \mathrm{NMR}$ (126 MHz, DMSO-d $)_{6} \delta 164.37$ (amide $\mathrm{C}=\mathrm{O}$ ), 161.82 (C-2), 147.51, 145.77, 145.34, 141.24, $139.54,139.09,129.93,125.40,121.06,120.18$, $118.92,118.13,116.77,115.07,113.24$, and 104.46 (aromatic and vinyl), $18.31\left(4-\mathrm{CH}_{3}\right)$.

\section{7-N-(3-methoxy-4-hydroxycinnamoyl)-amino-4- methylquinolin-2(1H)-one (17)}

The title compound was obtained in $84 \%$ yield, starting from 15 ; m.p. $327^{\circ} \mathrm{C}$; 
${ }^{1} \mathrm{H}$ NMR (500 MHz, DMSO-d 6 ) $\delta 11.57$ (s, 1H, H-1), 10.34 (s, 1H, 7-NH), 9.56 (s, 1H, 4'-OH), 7.86 $(\mathrm{d}, J=2.0 \mathrm{~Hz}, 1 \mathrm{H}, \mathrm{H}-8), 7.65(\mathrm{~d}, J=8.8 \mathrm{~Hz}, 1 \mathrm{H}, \mathrm{H}-$ 5), 7.52 (d, $J=15.6 \mathrm{~Hz}, 1 \mathrm{H}, \beta$ vinyl-H), 7.43 (dd, $J$ $=8.8,2.0 \mathrm{~Hz}, 1 \mathrm{H}, \mathrm{H}-6), 7.19(\mathrm{~d}, J=1.9 \mathrm{~Hz}, 1 \mathrm{H}, \mathrm{H}-$ 2'), 7.08 (dd, $J=8.3,1.9 \mathrm{~Hz}, 1 \mathrm{H}, \mathrm{H}-6$ '), 6.83 (d, $J=$ $8.1 \mathrm{~Hz}, 1 \mathrm{H}, \mathrm{H}-5$ '), 6.67 (d, $J=15.6 \mathrm{~Hz}, 1 \mathrm{H}, \alpha$ vinyl$\mathrm{H}), 6.25$ (dd, $J=1.7,1.2 \mathrm{~Hz}, 1 \mathrm{H}, \mathrm{H}-3), 3.83$ (s, 3H, methoxy $\left.-\mathrm{CH}_{3}\right), 2.38\left(\mathrm{~d}, J=1.1 \mathrm{~Hz}, 3 \mathrm{H}, 4-\mathrm{CH}_{3}\right) ;{ }^{13} \mathrm{C}$ NMR (126 MHz, DMSO-d ${ }_{6}$ ) $\delta 164.28$ (amide $\mathrm{C}=\mathrm{O}$ ), 162.02 (C-2), 148.81, 147.88, 147.60, 141.19, $139.53,126.09,125.34,122.11,118.93,118.56$, $115.73,115.50,113.53,110.86$ and 104.39 (aromatic and vinyl), 56.02 and 55.51 (methoxy $-\mathrm{CH}_{3}$ ), 18.56 and $18.39\left(4-\mathrm{CH}_{3}\right)$.

\section{BIOLOGICAL METHODOLOGY}

\section{Reagents}

Synthesized compounds were dissolved in DMSO (Sigma-Aldrich) to obtain $50 \mathrm{mM}$ stock solutions and kept in $4^{\circ} \mathrm{C}$ prior to use. Stock solutions were diluted in cell culture medium to obtain a required concentration of compounds $(5,50$ and 500 $\mu \mathrm{mol} / \mathrm{L})$. The final concentration of DMSO never exceeded $2 \%$.

\section{Cell culture}

Novel derivatives were tested in four cell lines: NIH3T3 (mouse fibroblasts), B16F10 (mouse melanoma), HeLa (human cervical cancer) and HL60 (human leukemia). Cells were maintained under standard conditions ( $95 \%$ humidity, $5 \% \mathrm{CO}_{2}, 37^{\circ} \mathrm{C}$ ) and cultured in an appropriate culture medium (DMEM for NIH3T3, B16F10, HeLa and RPMI for HL-60). The media were supplemented with $10 \%$ fetal bovine serum and $1 \%$ antibiotic-antimycotic solution (Sigma-Aldrich). Cells were passaged three times a week using $0.25 \%$ trypsin solution (SigmaAldrich). All cell lines were received from the American Type Culture Collection (ATCC).

\section{Cytotoxicity assays}

Crystal violet viability test was used to determine cytotoxic/cytostatic effects induced by novel derivatives (13). Cells were seeded on 96-well plates (Nest, 10x10 $10^{3}$ cells per well). After overnight incubation examined compounds $(0.5 \mathrm{ml})$ were added to cell culture in the range of concentrations (5, 50 and $500 \mu \mathrm{mol} / \mathrm{L})$. Cells were treated for $48 \mathrm{~h}$. After that time the viability test was performed as described in (14). Absorbance was measured at $\lambda=$ $570 \mathrm{~nm}$.
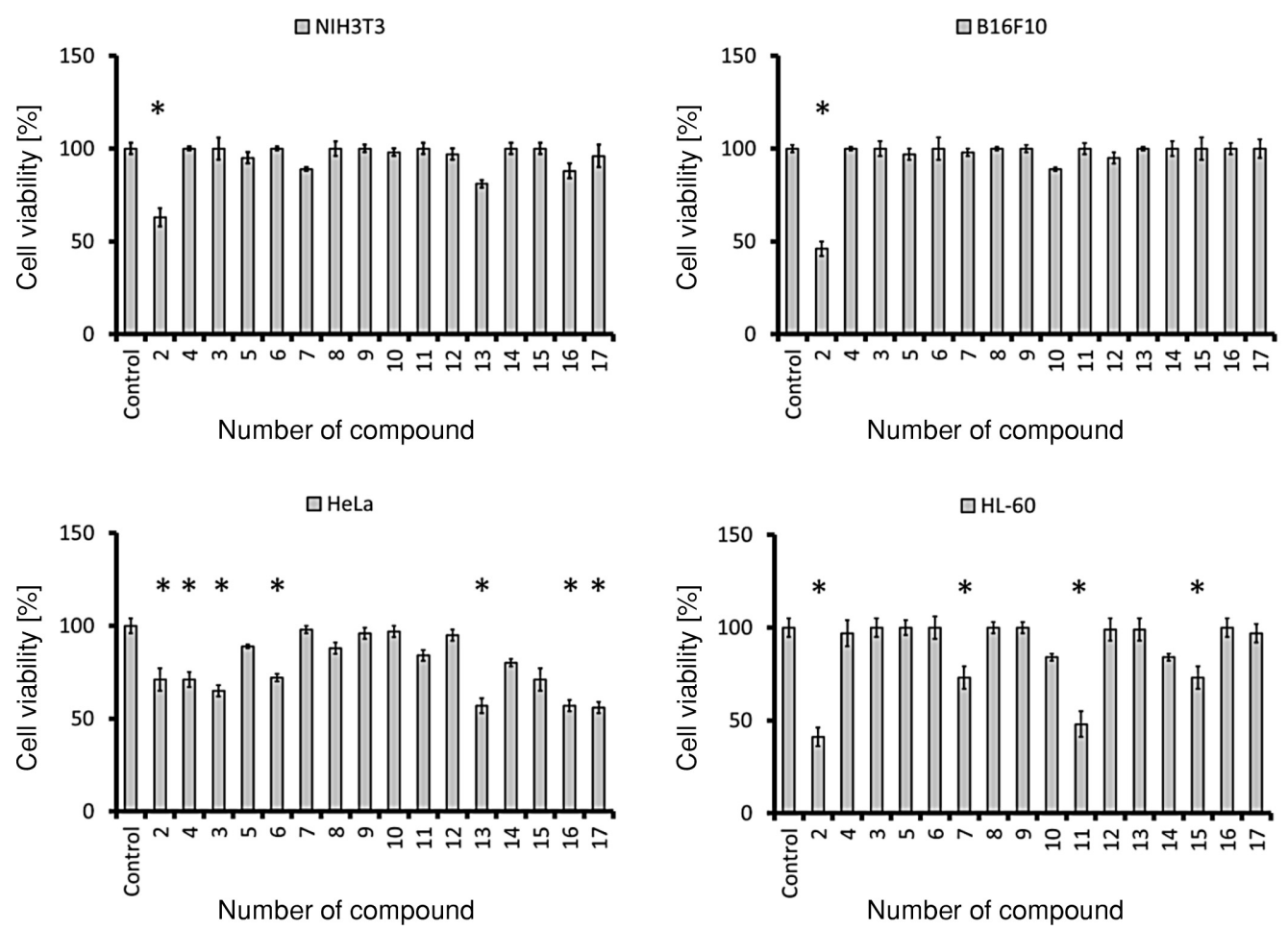

Figure 2. Cell viability test for $50 \mu \mathrm{mol} / \mathrm{L}$ concentration. NIH3T3 cells, B16F10 cells, Hela cells, HL-60 cells. * Significantly different from the controls ( $p<0.05$ in Student's t-test) 

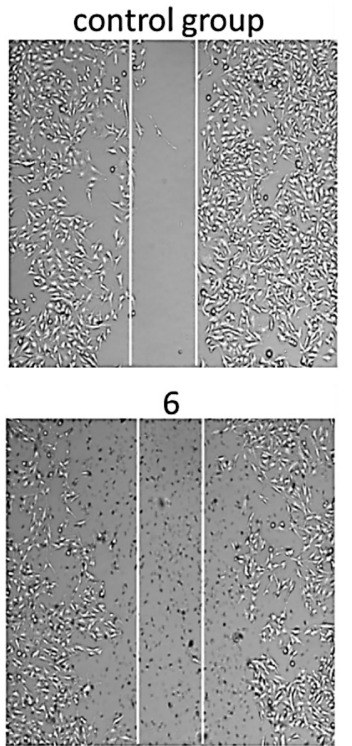

2

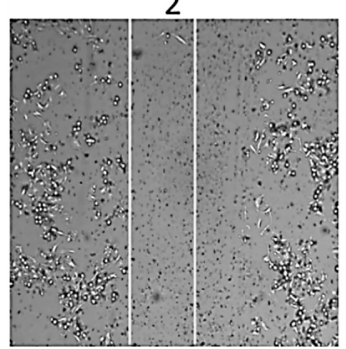

13

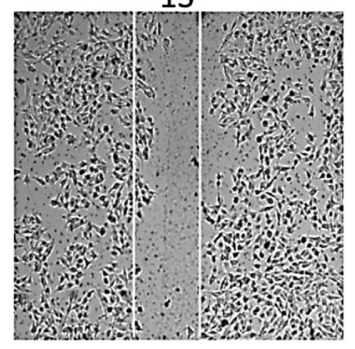

4

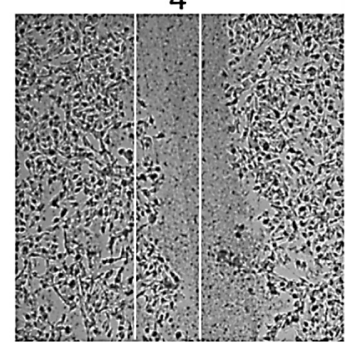

16

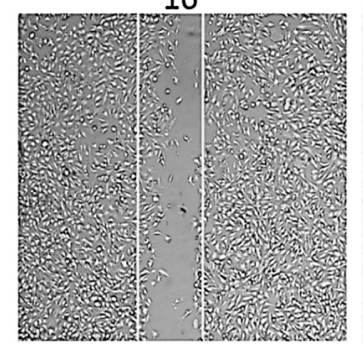

3

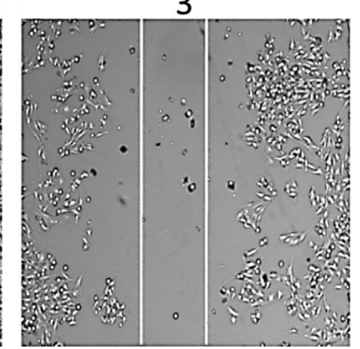

17

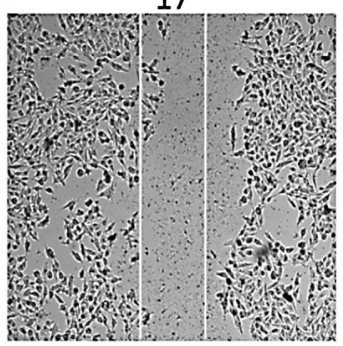

Figure 3. Photos of wound healing test on HeLa cells for $50 \mu \mathrm{mol} / \mathrm{L}$ concentration after 24 hours of incubation

\section{Wound healing assay}

The effects of novel compounds on cell migration were evaluated by wound healing assay (15). Tests were performed for selected compounds displaying the highest activity. Cells were seeded on 24-well plates (Falcon) and grown until approximately $60 \%$ confluency was reached prior to experimental treatment. To each well $0.5 \mathrm{~mL}$ of tested compound solution was added $(50 \mu \mathrm{mol} / \mathrm{L})$. After that, straight scratches were made using a pipette tip as a simulation of a wound. Images for the 0-time point were taken. After $24 \mathrm{~h}$ of incubation with compounds, the test was terminated and the endpoint images were taken. The results of the assay were visualized using phase contrast microscopy (JuLi, NanoEnTek).

\section{Statistical analysis}

In the statistical analysis Student's $t$-test was used to evaluate viability data. Test results were considered significant at a $p$-value $<0.05$

\section{RESULTS AND DISCUSSION}

\section{Cytotoxicity assays}

We studied time and dose-dependent effects of synthesized compounds on the viability of tumor and non-tumor cells as described in Material and Methods. Normal mouse fibroblasts (NIH3T3 cells) which served as a control of tumor cell selectivity for examined compounds, were resistant towards all examined substances except for component $\mathbf{2}$ that induced their significant growth retardation (Fig. 2). The examined tumor cell lines demonstrated various growth retardation under the influence of tested compounds. Mouse melanoma cells were affected by compound 2 . Other tested substances did not significantly alter the growth of B16F10 cells.

In contrast to B16F10 cells, HeLa cervical cancer cells were sensitive towards most novel compounds. Significant reduction of viability was demonstrated for compounds $2,4,3,6,13,16$ and 17. However, reduction of HeLa cells viability never exceeded $50 \%$ (Fig. 2).

Human leukemia cells were sensitive against compounds 2,7,11 and 15. Moreover, for compounds $\mathbf{2}$ and $\mathbf{1 1}$ viability reduction exceeding 50\% was obtained (Fig. 2)

These results show that compounds $4,3,6,7$ $11,13,15,16,17$ are selective for certain tumor cell lines. Substances with highest cytostatic/cytotoxic activity were selected for further tests.

\section{Wound healing assay}

Wound healing test was performed to evaluate the potential effect of examined compounds on cell migration. According to protocol, cells were seeded a plate and then the scratch on monolayer was made. Prepared model of a wound was exposed to examined compounds $(50 \mu \mathrm{mol} / \mathrm{L})$ at $24 \mathrm{~h}$. Endpoint size of a wound in the treated group was compared with a result for endpoint size of wound in untreated cells. The results are presented in Figure 3. White lines on micrographs shows the frame of a wound in 
the control group after 24 hours of incubation. Compounds 4, 13, 16 and 17 do not inhibit cell migration. However, the morphological changes and decreasing confluence of cells treated with 13 and 17 were observed. Compounds 2, 3 and $\mathbf{6}$ inhibited cell migration after 24 hours of incubation. In these groups decrease in a number of adherent cells was also observed.

\section{CONCLUSION}

In conclusion, we obtained sixteen new derivatives of 7-amino-4-methylquinolin-2(1H)-one and employed a unique methodology of synthesis of the substances. We developed the multistep synthesis of amides of 7-amino-4-methylquinolin-2(1H)-one derivatives. We have obtained sixteen novel compounds showing many structural similarities with drugs that are widely used as anticancer medications. Their structures were confirmed by ${ }^{1} \mathrm{H}$ and ${ }^{13} \mathrm{C}$ NMR spectra.

In vitro tests for a series of 16 novel compounds were performed. Their biological activity was tested in tumor models and in non-cancer cells. The results of the performed tests showed that some examined compounds are selective for cancer cells, but their activity for various types of cancer is different. Mouse melanoma cells were resistant to tested compounds, but human leukemia cells and human cervix cancer cells were sensitive for some of them. Seven compounds were selected for further test of cell migration inhibition in HeLa cells. Three of them presented the ability to inhibit cells migration.

\section{REFERENCES}

1. Siegel R.L., Miller K.D., Jemal A.: CA Cancer J. Clin. 65, 5 (2015).

2. Torre L.A., Bray F., Siegel R.L., Ferlay J., Lortet-Tieulent J., Jemal A.: CA Cancer J. Clin. 65, 87 (2015).

3. Baccarani M., Deininger M.W., Rosti G., Hochhaus A., Soverini S. et al.: Blood. 122, 872 (2013).

4. Elisei R., Schlumberger M.J., Müller S.P., Schöffski P., Brose M.S. et al.: J. Clin. Oncol. 31, 3639 (2013).

5. Singh H., Brave M., Beaver J.A., Cheng J., Tang S. et al.: Clin. Cancer Res. 23, 330 (2016).

6. Punnoose E.: Fox03a as predictive biomarker for pi3k/akt kinase pathway inhibitor efficacy. Patent WO2011130654 A1 (2011).

7. Anderson A.C., Wright D.L.: Expert Opin. Ther. Pat. 24, 687 (2014).

8. Fry D.W., Jackson R.C.: Cancer Metastasis Rev. 5, 251 (1987).

9. Sroka Z., Cisowski W.: Food Chem. Toxicol. 41, 753 (2003).

10. Gomes C.A., Girăo da Cruz T., Andrade J.L., Milhazes N., Borges F., Marques MPM.: J. Med. Chem. 46, 5395 (2003).

11. Hejchman E., Taciak P., Kowalski S., Maciejewska D., Czajkowska A. et al.: Pharmacol Rep. 67, 236 (2015).

12. Shiroya U., Patel M.: Med. Chem. Res. 22, 5227 (2013).

13. Feoktistova M., Geserick P., Leverkus M.: Cold Spring Harb. Protoc. 4, 343 (2016)

14. Młynarczuk-Biały I., Roeckmann H., Kuckelkorn U., Schmidt B., Umbreen S. et al.: Cancer Res. 66, 7598 (2006).

15. Liang C-C., Park A.Y., Guan J-L.: Nat. Protoc. 2, 329 (2007).

Received: 04.06. 2017 